\title{
NOVOS MINERAIS RECENTEMENTE APROVADOS PELA COMISSÃO DE NOVOS MINERAIS E NOMES DE MINERAIS DA ASSOCIAÇÃO MINERALÓGICA INTERNACIONAL (CNMNM - IMA)
}

\author{
JOSEPH A. MANDARINO*
}

Tradução de Daniel Atencio**

As informações a seguir são fornecidas pela CNMNM IMA para fins comparativos e como serviço aos mineralogistas que estão trabalhando com espécies noyas. Deseja-se que listas futuras sejam incluídas, a cada quatro ou seis meses, nas principais revistas que publicam trabalhos mineralógicos.

Cada mineral é descrito no seguinte formato:

\section{№ IMA}

(relações com outros minerais)

Fórmula química

Sistema cristalino, grupo espacial parâmetros da cela unitária

Transparência; brilho; cor.

Propriedades ópticas.

Linhas mais fortes no espectro de difração de raios $\mathrm{X}$.

Os nomes destas espécies aprovadas são considerados como informações confidenciais até que os autores tenham publicado suas descrições ou eles mesmos tenham divulgado as informações.

Nenhuma outra informação será forecida pela comissão.

\section{OS MINERAIS SEGUINTES FORAM APROVADOS EM 1990}

№ IMA: 90-002

(Ce,La) $\mathrm{Al}_{2} \mathrm{~B}_{3} \underline{\mathrm{O}}_{9}$

Hexagonal, $\mathrm{P} 62 \mathrm{~m}$

$$
\text { a } 4,610, \text { c } 9,358 \AA
$$

Transparente a translúcido; vítreo; amarelo claro.

Uniaxial $(+), \omega 1,703, \varepsilon 1,711$.

$3,67(100), 3,04(100), 2,458(75), 2,308(50), 2,020(50)$,

$1,953(50), 1,855(50), 1,835(50)$.

№ IMA: 90-004

Análogo da allanita-(Ce) com $\mathrm{Mg}$ dominante

$\mathrm{Ca}(\mathrm{Ce}, \mathrm{La}) \mathrm{MgAl}_{2} \mathrm{Si}_{3} \mathrm{O}_{12}(\mathrm{OH})$

Monoclínico, $\mathrm{P}_{1} / \mathrm{m}$ a 8,916 , b 5,700, c $10,140 \AA, \beta 114,72^{\circ}$

Transparente; vítreo; marrom amarelado claro em seção delgada.

Biaxial (+), $\alpha$ 1,735, ß 1,741, $\gamma$ 1,758, 2V(med.) $64^{\circ} ; 2 \mathrm{v}$ (calc.) $62^{\circ}$.

$9,1(40), \quad 3,50(50), \quad 2,910(90), \quad 2,842(50), \quad 2,698(100)$,

2,622(60), 2,177(40), 2,137(40).

\section{№ IMA: 90-005}

$\mathrm{Ca}_{5} \mathrm{Si}_{6}(0, \mathrm{OH})_{18 .} .5 \mathrm{H}_{2} \mathrm{O}$

Monoclínico, $\mathrm{Cc}$ ou $\mathrm{C} 2 / \mathrm{c}$

a 11,331 , b 7,353, c 22,67 $\AA$, p 96,59

Transparente; vítreo; incolor a branco.

Biaxial (-), $\alpha 1,575, \beta 1,580, \gamma$ 1,585, $2 \mathrm{~V}$ (calc.) $89,8^{\circ}$.
$11,25(100), 3,745(36), 3,304(51), 3,068(45), 3,034(60)$, $3,012(37), 2,811(41), 2,794(60)$.

№- IMA: $90-007$

Análogo da braunita e da neltnerita com $\mathrm{Cu}$ dominante $\mathrm{Cu}^{2+} \mathrm{Mn}_{6}^{+3}\left(\mathrm{O}_{8} / \mathrm{SiO}_{4}\right)$

Tetragonal, I4i/acd a 9,409, c $18,600 \AA$

Opaco; metálico; preto.

Em luz refletida: cinza, anisotropia muito fraca, fraca birreflectância, não-pleocróico.

R (min. - máx.): (20,8-21,2\%) 470 nm, (19,6-20,0\%) 546 $\mathrm{nm},(19,2-19,7 \%) 589 \mathrm{~nm},(18,7-19,2 \%) 650 \mathrm{~nm}$.

$2,703(100), 2,352(14), 2,135(16), 1,6516(30), 1,4167(10)$, $1,4023(12)$.

\section{№ IMA: 90-008}

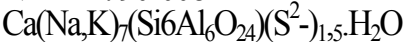

Hexagonal (trigonal), P31c a $12,855, \mathrm{c} 10,700 \AA$

Transparente; vítreo; amarelo.

Uniaxial (-), $\omega 1,584, \varepsilon 1,660$.

$4,824(70), 3,919(80), 3,720(100), 3,313(90), 2,694(35)$, $2,676(70), 2,471(35)$.

№ IMA: 90-009

$(\mathrm{Na}, \mathrm{Ca}, \mathrm{K})_{8}\left(\mathrm{Si}_{\mathrm{c}} \mathrm{Al} 6024\right)\left(\mathrm{SO}_{4}\right) 2 \mathrm{CLO}, 5 \mathrm{H}_{2} \mathrm{O}$

Hexagonal, $\mathrm{P}_{2} 22$

a $12,843, \mathrm{c} 32,239 \AA$

Transparente; vítreo; verde a amarelo-esverdeado.

Uniaxial $(+), \omega 1,528, \varepsilon 1,543$.

$4,84(40), 3,711(100), 3,314(80), 3,035(20), 2,988(16)$, 2,687(25), 2,470(16), 2,139(25).

№ IMA: $90-010$

$\mathrm{Fe}_{8-2 \mathrm{x}}\left[\left(\mathrm{As}_{1^{-} \mathrm{x}}, \mathrm{S}_{\mathrm{x}}\right) \mathrm{O}_{4}\right]_{6}(\mathrm{OH})_{6} .5 \mathrm{H}_{2} \mathrm{O}$, $\mathrm{x}$ aproximadamente 0,2

Ortorrômbico, $\mathrm{Pbcm}$ a 6,412 , b 19,45 , c $8,941 \AA$

Transparente a translúcido; gorduroso; laranja- cád mio Biaxial (-), $\alpha$ 1,94, ß 2,05, $\gamma 2$ 2,06, 2V(med.) $5^{\circ}, 2 \mathrm{~V}$ (calc.) $32^{\circ}$. 9,75(10), 4,476(4), 3,208(9), 3,047(5), 2,680(4), 2,153(4), $1,604(4)$.

№ IMA: 90-011

$\mathrm{HgAg}(\mathrm{Cl}, \mathrm{Br}, \mathrm{I}) \mathrm{S}$

Ortorrômbico, $\mathrm{P} 22_{1} 2$ a 6,803 , b 12,87, c $4,528 \AA$

Translúcido a opaco; subadamantino a submetálico; preto. Biaxial (provavelmente-), $\alpha \sim 2,2, \gamma \sim 2,3$.

$6,43(40), 3,762(60), 3,637(60), 3,283(30), 2,664(100)$, $2,265(40), 2,047(20)$.

\footnotetext{
* Presidente da Comissão de Novos Minerais e Nomes de Minerais (CNMNM) da Associação Mineralógica Internacional (IMA), Division of Minerais and Geochemistry CSIRO, Private Bag, P.O.; Wembley, W.A. Austrália 6014

** Representante Brasileiro da CNMNM - IMA, Departamento de Mineralogia e Petrologia, Instituto de Geociências, Universidade de São Paulo, Caixa Postal 20899, CEP 01498, São Paulo, SP, Brasil
} 
№ IMA: 90-012

$\mathrm{Na}_{6} \mathrm{~K}_{2}\left(\mathrm{Si}_{6} \mathrm{Al}_{6} \mathrm{O}_{24}\right)\left(\mathrm{SO}_{4}\right) \cdot 2 \mathrm{H}_{2} \mathrm{O}$

Hexagonal, $\mathrm{P}_{3}$

a 22,121, c 5,221

Transparente; vítreo; incolor.

Uniaxial (-), $\omega 1,508, \varepsilon 1,506$.

6,39(forte), 4,77(muito forte), 3,69(médio), 3,27(muito forte),

2,769(médio), 2,650(médio).

№ IMA: $90-013$

$\mathrm{Na}_{7}\left[\mathrm{Al}_{5} \mathrm{Si}_{7} \mathrm{O}_{24}\right] \mathrm{CO}_{3} .3 \mathrm{H}_{2} \mathrm{O}$

Hexagonal, $\mathrm{P}_{3} \mathrm{mc}$ a 12,575 , c 5,105 $\AA$

Transparente; vítreo; lilás escuro a claro.

Uniaxial (-), $\omega$ 1,509, \& 1,490.

$6,30(70), 4,61(50), 3,65(90), 3,22(100), 2,722(50), 2,597(20)$,

2,402(20), 2,097(20).

No IMA: 90-014

$\mathrm{Na}_{8}\left[\mathrm{Al}_{6} \mathrm{Si}_{6} \mathrm{O}_{24}\right](\mathrm{OH})_{2} \cdot 2 \mathrm{H}_{2} \mathrm{O}$

Hexagonal, $\mathrm{P}_{3}$ a 12,74, c $5,183 \AA$

Transparente; vítreo; azul claro ou incolor.

Uniaxial $(+) ; \omega 1,494, \varepsilon 1,501$.

6,43(25), 4,70(60), 3,68(70), 3,26(100), 2,756(50), 2,433(30).

\section{№ IMA: $90-015$}

$\mathrm{Na}_{3}(\mathrm{Y}, \mathrm{REE})\left(\mathrm{CO}_{3}\right)_{3} .3 \mathrm{H}_{2} \mathrm{O}$

Ortorrômbico, grupo espacial desconhecido, retículo primitivo a 10,136 , b 17,348, c 5,970

Transparente; vítreo a fosco; incolor.

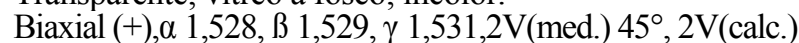

$71^{\circ}$.

6,53(55), 5,05(50), 4,85(65), 2,858(70), 2,597(50), 2,229(50), $2,076(100)$.

\section{№ IMA: 90-016}

Polimorfo ortorrômbico da natisita

$\mathrm{Na}_{3} \mathrm{TiSiO}_{5}$

Ortorrômbico, Pmma a 9,827 , b 9,167, c 4,799 $\AA$

Translúcido; adamantino; amarelo, amarelo-laranja emarromlaranja.

Biaxial (+), $\alpha$ 1,740, $\beta 1,741, \gamma 1,765,2 \mathrm{~V}\left(\right.$ med.) $20^{\circ}, 2 \mathrm{~V}($ calc.)

$23^{\circ}$.

$2,748(100), 2,257(25), 1,720(30), 1,680(30), 1,475(33)$, $1,443(35)$.

\section{№ IMA: 90-018}

Interestratificação regular 1:1 de cookeíta e paragonita

$\mathrm{Li}_{0,5} \mathrm{Na}_{0,5} \mathrm{Al}_{3} \mathrm{Si}_{3} \mathrm{ALO}$

Monoclínico, $\mathrm{C} 2 / \mathrm{m}$ a 5,158, b 8,914, c 23,83 $\AA$, p 94,23

Transparente; perláceo; branco.

Biaxial (-), $\alpha 1,58<<1,59$, ß 1,58 $<<1,59, \gamma 1,59<<1,60$,

$2 \mathrm{~V}$ (med.) $30-50^{\circ}$.

$11,89(70), 4,456(90), 4,325(90), 2,547(100), 2,476(70)$, $1,486(90)$.

\section{№ IMA: 90-019}

Análogo da calcofanita com $\mathrm{Mg}$ dominante

$(\mathrm{Mg}, \mathrm{Mn}, \mathrm{Ca}) \mathrm{Mn}_{3}{ }^{4+} \mathrm{O}_{7} .3 \mathrm{H}_{2} \mathrm{O}$

Triclínico, $\mathrm{Pi}$

a 7,534, b 7,525, c 8,204 $\AA, \alpha 89,753^{\circ}, \gamma 117,375^{\circ}, \gamma$ $120.000^{\circ}$

Opaco; fosco; preto café.

Em luz refletida: cinza, anisotropia clara, birreflectância fraca, não pleocróico.

Valores de R: $(23,0 \%) 470 \mathrm{~nm}, \quad(19,9 \%) 546 \mathrm{~nm}$, $(19,1 \%) 589 \mathrm{~nm},(18,6 \%) 650 \mathrm{~nm}$.
6,965(100), 5,539(3), 4,086(4), 3,522(3), 3,483(11), 2,230(8).

№ IMA: 90-020

$\mathrm{MnSO}_{3} .3 \mathrm{H}_{2} \mathrm{O}$

Ortorrômbico, Pnma a 9,762 , b 5,639, c 9,558

Transparente; vítreo; incolor. .

Biaxial $(+), \alpha 1,590, \beta 1,596, \gamma 1,636,2 \mathrm{~V}$ (med.) $41^{\circ}$, $2 \mathrm{~V}$ (calc.) $43^{\circ}$.

6,83 (forte), 4,33 (muito forte), 3,43 (muito forte), 2,704 (médio), 2,666 (médio), 2,414 (médio), 1,726 (médio).

№ IMA: 90-021

Análogo da lavenita com Ti dominante.

$\mathrm{NaCa}(\mathrm{Mn}, \mathrm{Fe})(\mathrm{Ti}, \mathrm{Nb}, \mathrm{Zr}) \mathrm{Si}_{2} \mathrm{O}_{7} \mathrm{OF}$

Monoclínico; $\mathrm{P} 2 \mathrm{i} / \mathrm{a}$

a 10,828, b 9,790, c 7,054 $\AA$, p 108,20

Translúcido a transparente; vítreo; marrom alaranjado, amarelo.

Biaxial (-), $\alpha$ 1,743, $\beta 1,785, \gamma 1,810,2 \mathrm{~V}$ (med.) $72-84^{\circ}$,

$2 \mathrm{~V}$ (calc.) $74^{\circ}$.

$3,942(20), 3,234(30), 2,859(100), 2,807(70), 1,762(20)$,

$1,741(20), 1,727(20), 1,688(20), 1,627(20)$.

№ IMA: $90-023$

$3 \mathrm{UO}_{3} \cdot 2 \mathrm{SeO}_{2} \cdot 7 \mathrm{H}_{2} \mathrm{O}$

Ortorrômbico, Pnc2 ou Pncm

a 8,025 , b 17,43 , c $6,935 \AA$

Translúcido a transparente; vítreo; amarelo vivo.

Biaxial (-), $\alpha$ 1,618, $\beta$ 1,738, $\gamma$ 1,765,2V(med.) $43^{\circ}, 2 \mathrm{~V}$ (calc.) $48^{\circ}$.

$8,01(100), \quad 4,01(70), \quad 3,468(60), \quad 3,186(50), \quad 3,119(70)$,

$2,912(80), 2,471(40)$.

№ IMA: $90-024$

Análogo da fenaksita com $\mathrm{Mn}$ dominante

$\mathrm{NaKMnSi}_{4} \mathrm{O}_{10}$

Triclínico, Pi a 6,993, b 8,219, c $10,007 \AA$, a $105,11^{\circ}$, p $100,76^{\circ}$, y

$114,79^{\circ}$

Transparente; vítreo; incolor a creme-rosado claro.

Biaxial (-), $\alpha$ 1,540, $\beta 1,551, \gamma 1,557,2 \mathrm{~V}$ (med.) $73^{\circ}, 2 \mathrm{~V}$ (calc.) $72^{\circ}$.

6,89(70), 3,45(100), 3,26(90), 3,05(80), 2,880(70), 2,715(70), $2,463(70)$.

№ IMA: 90-025

$\mathrm{Na}_{1}-\mathrm{Ca}_{3} \mathrm{Mg}(\mathrm{Ti}, \mathrm{Mn})_{4}\left(\mathrm{Si}_{2} \mathrm{O}_{7}\right)_{2}\left(\mathrm{PO}_{4}\right)_{6} \mathrm{O}_{3} \mathrm{~F}_{5}$

Triclínico, PI

a 5,412 , b 7,079, c $26,56 \hat{A}, \alpha 95,21^{\circ}, \beta 93,51^{\circ}, \gamma 90,10^{\circ}$

Translúcido a transparente; vítreo a perláceo; marrom claro.

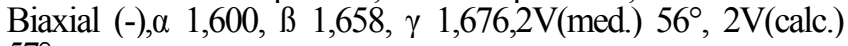
$57^{\circ}$.

$2,937(10), \quad 2,702(9), \quad 2,659(8), 2,048(8 B), \quad 1,771(513)$,

$1,730(5)$.

№ IMA: 90-026

$\mathrm{Na}_{14} \mathrm{CaMgTi}_{4}\left(\mathrm{Si}_{2} \mathrm{O}_{7}\right)_{2}\left(\mathrm{PO}_{4}\right)_{4} \mathrm{O}_{4} \mathrm{~F}_{2}$

Triclínico, $\mathrm{Pi}$

a 5,415 , b 7,081 , c $20,34 \hat{A}$, a $86,85^{\circ}, 094,40^{\circ}$, y $89,94^{\circ}$

Translúcido a transparente; vítreo a perláceo; marrom claro.

Biaxial (-), $\alpha$ 1,630, $\beta 1,678, \gamma$ 1,697,2V(med.) $62^{\circ}, 2 \mathrm{~V}$ (calc.)

$63^{\circ}$.

$2,880(10), 2,702(8 \mathrm{~B}), 2,636(7), 2,050(5), \quad 1,662(4 \mathrm{~B})$,

$1,600(5)$.

№ IMA: $90-027$

$(\mathrm{Ca}, \mathrm{Mn})_{4} \mathrm{Be}_{3} \mathrm{Si}_{6} \mathrm{O}_{17}(\mathrm{OH})_{4} 3 \mathrm{H}_{2} \mathrm{O}$

Ortorrômbico, grupo espacial desconhecido

a 8,724, b 23,14, c $4.923 \AA$ 
Translúcido; vítreo; branco a cinza claro ou bege.

Biaxial, índice de refração médio 1,604.

11,64(93), 5,80(68), 3,87(76), 3,16(74), 2,889(75), 2,837(100), 2,494(58).

\section{№ IMA: $90-028$}

$\mathrm{NaLiSi}_{2} \mathrm{O}_{5} .2 \mathrm{H}_{2} \mathrm{O}$

Monoclínico, A2/n

a 5,061, b 8,334, c $14.383 \AA, p$ 96,67

Transparente a opaco; vítreo a terroso; incolor a branco.

Biaxial (+), $\alpha$ 1,515, $\beta 1,516, \gamma$ 1,518,2V(med.) $64^{\circ}, 2 \mathrm{~V}$ (calc.) $71^{\circ}$.

$7,14(100), \quad 4,24(80), \quad 4,14(100), \quad 4,02(80), \quad 2,847(100)$, 2,698(50), 1,610(40), 1,557(40).

\section{№ IMA: $90-030$}

$\mathrm{NaLi}_{2} \mathrm{PO}_{4}$

Ortorrômbico, Pmnb a 6,884 , b 9,976, c 4,927

Transparente a translúcido; vítreo, incolor; branco, azul muito claro, amarelo muito claro.

Biaxial (-), $\alpha 1,533, \beta 1,540, \gamma$ 1,541,2V(med.) $49^{\circ}, 2 \mathrm{~V}$ (calc.) $41^{\circ}$.

4,020(100), 3,507(100), 3,441(100), 2,833(40), 2,712(40), 2,493(90), 2,462(90), 1,721(40).

\section{No $\quad$ IMA: $\quad 90-031$}

$\mathrm{Pb}_{3}\left(\mathrm{Fe}^{3+}, \mathrm{Mn}^{3+}\right){ }_{4} \mathrm{Mn}_{3}^{4+} \mathrm{O}_{15}$

Hexagonal, $\mathrm{P}_{3} / \mathrm{mcm}$

a $10,037, \mathrm{c} 13,67 \AA$

Opaco; metálico; preto.

Em luz refletida: branco vivo, forte anisotropia, birreflectância moderada, não-pleocróico.

$\mathrm{R}_{\mathrm{O}} \& \mathrm{R}_{\mathrm{E}}$ : (31,0-26,1\%) $470 \mathrm{nrn},(29,5-25,1 \%) 546 \mathrm{~nm},(28,5-$ $24,4 \%) 589 \mathrm{~nm},(27,2-23,4 \%) 650 \mathrm{~nm}$.

3,42(5), 3,18(8), 2,828(7), 2,663(10), 2,366(6), 1,687(8).

\section{№ IMA: $90-032$}

\section{$\mathrm{Mg}_{5} \mathrm{Ba}\left(\mathrm{PO}_{4}\right)_{4} 8 \mathrm{H}_{2} \mathrm{O}$}

Ortorrômbico, Pmma, Pmc2 1 ou Pma2 a 12,829 , b 8,335, c $18,312 \AA$

Transparente; vítreo a sedoso; marrom-amarelado à rosa claro. Biaxial $(+), \alpha 1,552, \beta 1,552, \gamma 1,558,2 \mathrm{~V}$ (med.) $23^{\circ}$, $2 \mathrm{~V}$ (calc.) $0^{\circ}$.

10,51(100), 3,874(32), 3,520(34), 3,081(78), 3,054(41), $2,969(44), 2,839(34)$

\section{№ IMA: $90-033$}

$\mathrm{Pb}_{4} \mathrm{Cu}_{4} \mathrm{Si}_{4} \mathrm{Oi}_{12}\left(\mathrm{HCO}_{3}\right)_{4} \mathrm{ClH}$

Tetragonal, I4/m

a 14,234 , c $6,103 \AA$

Transparente; vítreo; azul vivo.

Uniaxial $(+), \omega 1,786, \varepsilon 1,800$

$10,2(10), 5,644(7), 4,495(10), 3,333(10), 3,013(9), 2,611(5)$.

\section{№ IMA: 90-036}

$\mathrm{Cu}_{4} \mathrm{Al}_{2}\left[\mathrm{HSbO} 4, \mathrm{SO}_{4}\right](\mathrm{OH})_{10}\left(\mathrm{CO}_{3}\right) \cdot 2 \mathrm{H}_{2} \mathrm{O}$

Monoclínico, $\mathrm{P} 2_{1}$

$$
\text { a } 10,765 \text {, b 2,903, c } 12.527 \AA, p 95,61^{\circ}
$$

Transparente; sedoso; azul esverdeado.

Biaxial (+), $\alpha$ 1,626, $\beta 1,646, \gamma$ 1,682, $2 \mathrm{~V}$ (med.) $77^{\circ}, 2 \mathrm{~V}$ (calc.) $75^{\circ}$

$5,62(50), 5,160(90), 4,276(100), 3,565(40), 2,380(35)$, $2,326(35)$.

\section{No- IMA: 90-037}

$\mathrm{Cu}_{4}\left(\mathrm{UO}_{2}\right)\left(\mathrm{MoO}_{4}\right)_{2}(\mathrm{OH})_{6}$

Monoclínico, Al2l, Alml ou A12/ml a $5,529^{\wedge}$ b 6,112 , c $19.83 \AA$, p $103,9^{\circ}$

Transparente; vítreo a gorduroso; verde escuro a preto.
Biaxial (-), $\alpha$ 1,90, $\beta$ 1,93, $\gamma$ 1,96,2V(med.) $90^{\circ}, 2 \mathrm{~V}$ (calc.) $89^{\circ}$. $4,815(80), \quad 4,425(40), 4,276(40), 4,100(100), \quad 3,734(90)$, $3,254(40), 2,628(40), 2,482(60)$.

No MA: $90-040$

$\mathrm{Ca}_{3} \mathrm{Cu}_{5} \mathrm{Si}_{9} \mathrm{O}_{26}$

Monoclínico, $\mathrm{C} 2 / \mathrm{c}$ a 10,160 , b 10,001, c 19,973 $\AA$, p $91,56^{\circ}$

Transparente; vítreo; azul-esverdeado.

Biaxial $(+), \alpha \quad 1,722, \beta 1,723, y \quad 1,734,2 \mathrm{~V}$ (med.) $73^{\circ}$,

$2 \mathrm{~V}$ (calc.) $34^{\circ}$.

7,13(60)6,70(70), 3,12(90), 3,00(100), 2,45(60), 2,41(70).

№ IMA: 90-041

$\mathrm{Ca}_{3}\left(\mathrm{SO}_{3}\right)_{2} \mathrm{SO}_{4} \cdot 12 \mathrm{H}_{2} \mathrm{O}$

Hexagonal R3m a 11,350, c $28,321 \AA$

Transparente; vítreo; incolor.

Uniaxial $(+), \omega 1,4941, \varepsilon 1,4960$

$8,11(80), 5,73(100), 3,63(60), 3,28(40), 2,69(80), 2,11(40)$.

\section{$\mathrm{N}^{-}$IMA: $90-042$}

$\mathrm{Mn}(\mathrm{Mg}, \mathrm{Mn})_{2} \mathrm{Zn}_{2}(\mathrm{OH})_{10} .4 \mathrm{H}_{2} \mathrm{O}$

Monoclínico, $\mathrm{C} 2 / \mathrm{m}$

a 15,47, b 6,369, c 5,576 $\AA, 0101,29^{\circ}$

Principalmente opaco, mas também translúcido; vítreo a fosco ou terroso; marrom escuro.

Em luz refletida: cinza, anisotropia fraca, birreflectância muito fraca, não-pleocróico.

R(mín.-máx.): (8,54-8,65\%)470nm, $(8,07-8,23 \%) 546 \mathrm{~nm}$, $(8,00-8,19 \%) 589 \mathrm{~nm},(7,89-8,18 \%) 650 \mathrm{~nm}$.

7,61(10), 3,96(5), 3,45(3), 2,977(4), 2,745(6), 2,673(3).

№ IMA: $90-043$

Dimorfo monoclínico da mimetita

$\mathrm{Pb}_{5}\left(\mathrm{AsO}_{4}\right)_{3} \mathrm{Cl}$

Monoclínico, $\mathrm{P} 2 \mathrm{i} / \mathrm{b}$ a 10,189 , b 20,372, c 7,46 $\AA$, p $119,88^{\circ}$

Translúcido; resinoso; branco-amarelado.

Biaxial (-), $\alpha, \beta$ e $\gamma>1,8,2 \mathrm{~V}$ (med.) $8^{\circ}$.

$3,342(50), 3,048(100), 3,008(70), 2,947(70), 2,106(60)$, $1,961(50), 1,903(50)$.

№ IMA: $90-044$

$\mathrm{NaVO}_{3}$

Ortorrômbico, Pnma a 14,134, b 3,648, c 5,357

Transparente; sedoso; incolor.

Biaxial (+), $\alpha$ 1,780, $\beta 1,800, \gamma>1,85,2 \mathrm{~V}$ (med.) $30-40^{\circ}$.

$7,07(11), \quad 5,05(100), \quad 3,530(25), \quad 3,241(18), \quad 3,016(13)$, 2,957(35), 2,685(12).

№ IMA: 90-045

$\mathrm{Bi}_{2} \mathrm{Cu}_{3}(\mathrm{OH})_{2} \mathrm{O}_{2}\left(\mathrm{PO}_{4}\right)_{2} \cdot 2 \mathrm{H}_{2} \mathrm{O}$

Monoclínico, $\mathrm{C} 2 / \mathrm{m}$

a 12,358, b 6,331, c 9,060 А, p 122,70

Translúcido; vítreo; azul céu a azul vivo escuro.

Biaxial (-), ß 1,89, 2V(med.) $68^{\circ}$.

7,623(8), 6,093(6), 5,405(6), 5,201(7), 3,039(10), 2,921(9), 2,197(6).

\section{№ IMA: 90-047}

$\mathrm{Pt}_{5} \mathrm{Se}_{4}$

Monoclínico, $\mathrm{P} 2 \mathrm{i} / \mathrm{c}$ a $6,61, \mathrm{~b} 4,60$, c $11.10 \AA, \mathrm{p} 101,4^{\circ}$

Opaco; metálico; bronze escuro a preto.

Em luz refletida: branco, com tonalidade amarronzada, anisotropia muito forte, birreflectância muito forte, pleocroísmo fraco.

R(mín.-máx.): (35 ,2-54,8\%)470nm, (38,6-58,6\%)546nm, 
$(40,2-60,8 \%) 589 \mathrm{~nm},(42,4-63,2 \%) 650 \mathrm{~nm}$. 5,45(60), 3,27(60), 2,93(80), 2,78(60), 2,648(60B), 2,465(60), $1,875(100 \mathrm{~B}), 1,812(70)$.

\section{№ IMA: $90-048$}

PdBiSe

Cúbico, $\mathrm{P} 4.32$ ou $\mathrm{P}_{3} 32$ a $6,448 \AA$

Opaco; metálico; amarelo claro.

Em luz refletida: amarelo-rosado, não-anisotrópico, sem birreflectância, não-pleocróico.

Valores de R: $(47,5 \%) 470 \mathrm{~nm}, \quad(48,3 \%) 546 \mathrm{~nm}$, $(46,8 \%) 589 \mathrm{~nm},(45,6 \%) 650 \mathrm{~nm}$.

2,89(10), 2,63(9), 1,943(9), 1,724(5), 1,376(4).

№ IMA: 90-049

$\mathrm{CaBe}_{3}(\mathrm{OH})_{2}\left(\mathrm{PO}_{4}\right)_{2} 4 \mathrm{H}_{2} \mathrm{O}$

Monoclínico, $\mathrm{Ce}$ a 11,897 , b 9,707, c 9,633 $\AA$, p 95,76

Translúcido; vítreo; incolor.

Biaxial (+), $\alpha 1,5203, \beta 1,5205, \gamma 1,5300,2 \mathrm{~V}($ med. $)<10^{\circ}$, $2 \mathrm{~V}$ (calc.) $17^{\circ}$.

$5,92(60), \quad 4,33(50), \quad 3,421(70), \quad 2,959(60), \quad 2,945(45)$, $2,5130(100)$.

\section{$\mathrm{N}^{\mathrm{0}}$ IMA: 90-050}

Análogo da stilpnonelana com $\mathrm{Mn}$ dominante

$(\mathrm{K}, \mathrm{Na})_{4}\left(\mathrm{Mn}, \mathrm{Zn}, \mathrm{Mg}, \mathrm{Fe}^{3+}\right)_{48}(\mathrm{Si}, \mathrm{Al})_{72}(\mathrm{O}, \mathrm{OH})_{216} \mathrm{nH}_{2} \mathrm{O}$ (n aproximadamente 6)

Triclínico, $\mathrm{Pl}$ ou $\mathrm{P} \overline{\mathrm{l}}$ a 5,521, b 9,560, c 36,57 $\AA$ /cela orto-hexagonal.

Transparente a translúcido; vítreo; marrom escuro.

Biaxial (-), $\alpha$ 1,545, $\beta 1,583, \gamma \quad 1,583,2 \mathrm{~V}$ (med.) $10^{\circ}$, $2 \mathrm{~V}($ calc. $) 0^{\circ}$.

12,3(100), 2,737(30), 2,583(40), 2,362(30), 1,594(30), $1,580(30)$.

\section{№ IMA: $90-051$}

Membro do grupo da enigmatita

$(\mathrm{Ca}, \mathrm{Na})_{2}\left(\mathrm{Fe}^{2+}, \mathrm{Fe}^{3+}, \mathrm{Ti}\right)_{6}(\mathrm{Si}, \mathrm{Be}, \mathrm{A} 1)_{6} \mathrm{O}_{20}$

Triclínico, PI ou Pi a 10,385 , b 10,751, c $8,959 \AA, \omega 104,76^{\circ}$, p $97,03^{\circ}$, y $125,47^{\circ}$

Opaco a subtranslúcido; vítreo; preto.

Biaxial (-?), $\alpha 1,78, \gamma 1,82,2 \mathrm{~V}$ (med.) grande.

8,029(90), 3,122(46), 2,9243(59), 2,6756(48), 2,5291(100), 2,0993(63), 2,0758(47).

№ IMA: $90-052$

Análogo da escorodita e da mansfieldita com In dominante $\mathrm{In}\left(\mathrm{AsO}_{4}\right) \cdot 2 \mathrm{H}_{2} \mathrm{O}$

Ortorrômbico, Pcab a 10,45 , b 10,32, c $9,09 \AA$

Transparente; vítreo; verde claro a verde-amarelado.

Biaxial (-), n médio aproximadamente $1,65,2 \mathrm{~V}$ (med.) $55-76^{\circ}$. 5,719(70), 4,537(100), 4,162(40), 3,2461(80), 3,1073(80), $2,6568(50), 2,5426(45)$.

№ IMA: 90-054

$\left[(\mathrm{Na}, \mathrm{K})_{0} \mathrm{Cl}_{2}\right]\left(\mathrm{Ca}_{2} \mathrm{Cl}_{2}\right)\left(\mathrm{Si}_{6} \mathrm{Al}_{6} \mathrm{O}_{24}\right)$

Hexagonal, P6a ou Póa/m

a 25,771 , c $5,371 \AA$

Transparente; vítreo; incolor.

Uniaxial (+), $\omega 1,529, \varepsilon 1,532$

4,85 (forte), 3,71 (muito forte), 3,31 (muito forte), 2,788 (forte), 2,677 (médio), 2,474 (médio), 2,147 (médio), 1,804 (médio), 1,380 (médio).

№ IMA: 90-055

$(\mathrm{Pd}, \mathrm{Cu}, \mathrm{Fe})_{9} \mathrm{SnTe}_{2} \mathrm{~S}_{2}$

Tetragonal, grupo espacial des $\omega$ nhecido a 9,044, c 4,937

Opaco; metálico; cor megascópica desconhecida.

Em luz refletida: rosa-amarelado, anisotropia forte, birreflectância distinta a forte, pleocroísmo pronunciado.

R(mín.-máx.) (33,7-41,6\%) 470 nm, (38,5-48,7\%) 546 nm, $(40,4-51,8 \%) 589 \mathrm{~nm},(42,0-54,9 \%) 650 \mathrm{~nm}$.

2,472(10), 2,260(9), 2,022(6), 1,361(4), 1,213(5), 1,205(5), $1,129(5)$.

$\mathrm{N}^{\mathrm{0}}$ IMA: $90-056$

Análogo de $\mathrm{Fe}^{3+}$ da surita

$(\mathrm{Pb}, \mathrm{Ca})_{2 \cdot 3}\left(\mathrm{CO}_{3}\right)_{1,5-2}(\mathrm{OH}, \mathrm{F})_{0,5-1}\left[\left(\mathrm{Fe}, \mathrm{Al}_{2}\right)_{2} \mathrm{Si}_{4} \mathrm{O}_{10}(\mathrm{OH})_{2}\right] \cdot \mathrm{nH}_{2} \mathrm{O}$

Monoclínico, P2i ou P2i/m a 5,241, b 9,076, c 16,23 $\AA, \mid 390,03^{\circ}$

Transparente; sedoso; verde amarelado claro a verde floresta escuro.

Biaxial (+), a 1,757, ß 1,763, $\gamma 1,773,2 \mathrm{~V}$ (calc.) $76^{\circ}$

$16,1(40), 4,53(100), \quad 3,727(35), \quad 3,240(90), \quad 2,612(80)$, $2,272(50)$.

№ IMA: 90-057

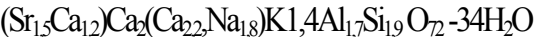

Hexagonal, Pós/mmc a 13,244 , c $15,988 \AA$

Transparente; vítreo; incolor.

Uniaxial (-), $\omega 1,522, \varepsilon 1,507$

6,58(80), 3,80(100), 2,95(70), 2,70(50), 2,50(50), 2,21(70), $1,83(50)$. 